\title{
A!
}

This is an electronic reprint of the original article.

This reprint may differ from the original in pagination and typographic detail.

Pöykkö, S.; Puska, M.J.; Alatalo, M.; Nieminen, R.M.

\section{Metastable defect complexes in GaAs}

Published in:

Physical Review B

DOI:

10.1103/PhysRevB.54.7909

Published: 15/09/1996

Document Version

Publisher's PDF, also known as Version of record

Please cite the original version:

Pöykkö, S., Puska, M. J., Alatalo, M., \& Nieminen, R. M. (1996). Metastable defect complexes in GaAs. Physical Review B, 54(11), 7909-7916. https://doi.org/10.1103/PhysRevB.54.7909

This material is protected by copyright and other intellectual property rights, and duplication or sale of all or part of any of the repository collections is not permitted, except that material may be duplicated by you for your research use or educational purposes in electronic or print form. You must obtain permission for any other use. Electronic or print copies may not be offered, whether for sale or otherwise to anyone who is not an authorised user. 


\title{
Metastable defect complexes in GaAs
}

\author{
S. Pöykkö, ${ }^{*}$ M. J. Puska, ${ }^{\dagger}$ M. Alatalo, ${ }^{\dagger}$ and R. M. Nieminen ${ }^{\S}$ \\ Laboratory of Physics, Helsinki University of Technology, FIN 02150 Espoo, Finland
}

(Received 9 February 1996; revised manuscript received 2 May 1996)

\begin{abstract}
We have studied the As-vacancy-Si-impurity and the As-vacancy-As-antisite complexes in GaAs using state-of-the-art electronic structure methods. The complexes show metastability as a function of the position of the impurity or the antisite atom similarly to the large-lattice relaxation models for the isolated $D X$ and EL2 centers. Our findings suggest the enlargement of the family of metastable defects in GaAs, and the results enlighten the metastability mechanisms in the large-lattice relaxation model. In order to discuss the possible experimental detection of this type of metastability, we calculate the positron states and annihilation characteristics for the defect complexes. [S0163-1829(96)02135-2]
\end{abstract}

\section{INTRODUCTION}

The most important native point defect in undoped GaAs crystals grown under As-rich conditions is the so-called EL2 center. In $\mathrm{Ga}_{1-x} \mathrm{Al}_{x} \mathrm{As}$ with a high $\mathrm{Al}$ concentration $(x>0.22)$, or in GaAs under high hydrostatic pressure the group-IV Ga site and group-VI As-site dopants form deep $D X$ centers. The EL2 and the $D X$ centers show interesting and important metastability. The widely accepted models by Chadi and Chang and Dabrowski and Scheffler for these metastabilities are based on large lattice relaxations (LLR's). ${ }^{1,2}$ For EL2 the stable state is formed by a neutral As antisite $\left[\left(\mathrm{As}_{\mathrm{Ga}}\right)^{0}\right]$ which, when excited, moves in the open [111] direction to an interstitial site leaving a Ga vacancy $\left(V_{\mathrm{Ga}}\right)$ behind [see Fig. 1(a)]. In the case of the $D X$ center in the singly negative charge state, the stable state corresponds to the interstitial site of the dopant atom in the [111] direction from the substitutional site.

According to the LLR models the metastability of the EL2 and $D X$ centers is controlled by the localized deep electron states in the band gap. ${ }^{1,2}$ In the case of the neutral EL2 and the singly negative $D X$ center, the deep levels are occupied by two electrons. In the substitutional configuration the deep states belong to the totally symmetric $a_{1}$ representation of the $T_{d}$ symmetry group. For the EL2 center in the interstitial configuration of $C_{3 v}$ symmetry, the displaced As atom is bonded to three neighboring As atoms forming locally a graphitelike $s p^{2}$-bonded system. In the interstitial-site configuration the deep-level electrons occupy a dangling bond at the As atom on the opposite side of the evolved Ga vacancy with respect to the displaced atom. The dangling bond points toward the center of the vacancy, and it is antibonding with respect to the displaced As atom. This stabilizes the LLR configuration. The electronic structure of the $D X$ center is similar to that of the EL2, but some qualitative differences exist, as will be discussed below. The displacement of the As atom in the EL2 center can be induced by electronic excitation, whereas that of the dopant atom in the $D X$ center takes place when the charge state of the defect changes (bistability).

In this work we consider defect complexes formed in GaAs by an As vacancy with an As antisite $\left(V_{\mathrm{As}} \mathrm{As}_{\mathrm{Ga}}\right)$ or with a $\mathrm{Si}$ dopant atom $\left(V_{\mathrm{As}} \mathrm{Si}_{\mathrm{Ga}}\right)$. These are maybe the sim- plest defect complexes involving an As antisite or a substitutional Si impurity. In the singly positive charge state $\left(V_{\mathrm{As}} \mathrm{As}_{\mathrm{Ga}}\right)^{+}$of the former, and the neutral charge state $\left(V_{\mathrm{As}} \mathrm{Si}_{\mathrm{Ga}}\right)^{0}$ of the latter, the defects have two electrons in the deep levels. We demonstrate that they show a metastability similar to the EL2 center. This means that there exist energy minima corresponding to the substitutional site neighboring the vacancy, and to the interstitial site further away in the [111] direction opposite to the vacancy [see Fig. 1(b)]. The stabilization of the latter configuration now has to differ, at least in details, from that of the isolated EL2 or $D X$ centers, because on the opposite side of the Ga vacancy created there is now no As atom to host a dangling bond.

The defect complexes studied enlarge the family of metastable defects. Thereby they serve alternatives for explaining the structures of defects observed ${ }^{3}$ to show metastability similar to but differing in details from that for isolated EL2 or $D X$ centers. This kind of defect may also appear after electron irradiation of GaAs samples. ${ }^{4}$ In the defects studied the metastability is connected with a change of the open volume at the core of the defect. Therefore this kind of meta- (a)

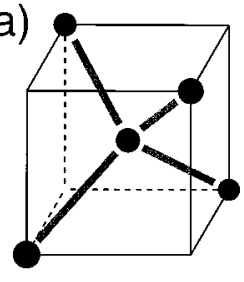

(b)

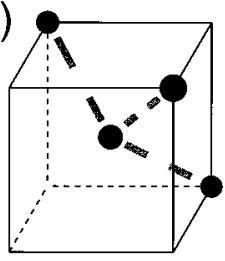

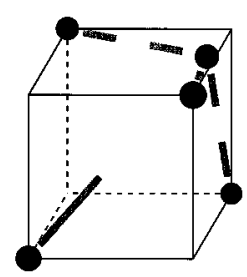

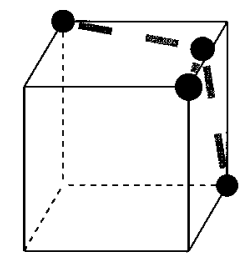

FIG. 1. Schematic views of (a) DX/EL2 centers and (b) vacancy impurity-antisite defects. The substitutional configurations are shown on the left-hand side, and the interstitial configurations on the right-hand side. The neighboring As atoms are shown as black circles, and the defect $\mathrm{Si}$-As atoms as gray circles. 
stability should be well detectable in positron annihilation experiments which have demonstrated the existence of the open volume (Ga vacancy) in the interstitial ionic configurations of the EL2 (Ref. 5) and DX (Ref. 6) centers. As a matter of fact, the positron lifetime for an As-vacancy-type defect has been found to depend strongly on the charge state of the defect. ${ }^{7}$ The strong charge state dependence could be a sign of a LLR similar to that between the stable and the metastable states of the EL2 or the DX centers. Below, we estimate the changes of the positron annihilation characteristics between the different configurations of the As vacancyantisite or vacancy-impurity complexes, and show that they are large enough to be detected in experiments.

We study the vacancy-antisite and vacancy-dopant complexes using first-principles electronic structure methods. ${ }^{8}$ Special care has been taken in order to achieve converged results with respect to approximations such as the supercell size, Brillouin-zone sampling, and the extent of the basisfunction set. First, we have made model calculations following Chadi and Chang or Dabrowski and Scheffler. In these calculations the atomic positions of an ideal lattice are assumed, and a vacancy is created by removing an As atom. A $\mathrm{Ga}$ atom neighboring the vacancy is substituted with an As or $\mathrm{Si}$ atom, which is then moved in the [111] direction away from the vacancy, so that the host atoms are not allowed to relax. Thereby we obtain the total energy of the system as a function of the ionic configuration. The ions have then been allowed to relax in the substitutional and interstitial-site configurations in order to show that the local-energy minima are not destroyed by the lattice relaxation.

\section{COMPUTATIONAL METHODS}

Our calculations are based on the density-functional theory (DFT). The electron exchange-correlation energy is calculated within the local-density approximation (LDA). ${ }^{9}$ We use first-principles norm-conserving pseudopotentials. ${ }^{10}$ These pseudopotentials are fully separable, ${ }^{11}$ and the $d$ components are used as local ones. For the plane-wave basis set, a high cutoff energy of $15 \mathrm{Ry}$ is used to ensure accurate results. The equilibrium lattice constant, according to our calculations, is $5.56 \AA$, which is slightly less than the experimental value of $5.65 \AA$. This discrepancy is typical for the LDA calculations. However, it is not expected to affect appreciably the results obtained, and we will use the theoretical lattice constant in our calculations. The defects are described in the supercell approximation using periodic boundary conditions.

We calculate the electronic structures using an efficient second-order damped dynamics method ${ }^{12}$ combined with the Williams-Soler algorithm. ${ }^{13}$ With respect to the electronic degrees of freedom, this method leads to a convergence, which is at least five times faster than the plain WilliamsSoler algorithm.

The atomic relaxations around a defect are calculated in a quasiadiabatic manner. This means that after each ionic movement the electronic structure is iterated toward the selfconsistency (adiabaticity) within a certain accuracy before the ions are moved again. The adiabaticity criterion for the electronic structure depends on the Hellmann-Feynman forces acting on the ions at their previous positions. The

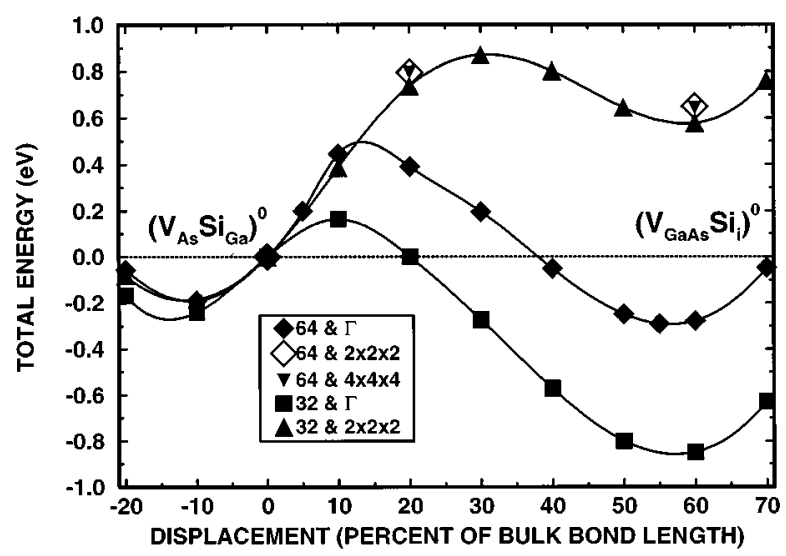

FIG. 2. Total energy of the neutral silicon-impurity-arsenicvacancy pair as a function of the Si-atom displacement from the Ga-atom substitutional position along the open [111] direction. Results from calculations with different supercell sizes and k-point samplings are shown (see the text). Atomic relaxations have not been allowed.

adiabaticity criterion is loose if the forces acting on the ions are large. This is the case in the beginning of the ionic relaxation, when the forces for the ions close to the center of the defect are large. The loose adiabaticity criterion introduces random components in the forces, but these components are small in magnitude in comparison with the forces of largest absolute values. The corresponding errors in the smaller forces for ions further away from the defect are relatively large but they are not crucial, since the ionic movements are there small. As a matter of fact small random components are useful, since they help the system to sample the phase space effectively. At the final stages of the ionic relaxation the forces acting on the ions are weak, leading to a stiff criterion for the adiabaticity. This means that the electronic structure is calculated very accurately, which ensures the reaching of the true ground state.

The use of the above-described quasiadiabatic method is possible because we want to find the minimum-energy configuration for each defect, and we do not want to make any dynamical studies. In the quasiadiabatic method a very accurate electronic structure has to be calculated only when it is really needed, i.e., when the ions are close to their configuration of minimum energy. An accurate electronic structure for the starting configuration is not needed. As a result, in a typical defect calculation the number of time steps needed to calculate the accurate electronic and ionic structures with this procedure is only $2-4$ times the number of time steps needed to calculate the electronic structure for fixed ionic positions.

The effect of the supercell size has been studied by the use of supercells corresponding to 32- and 64-atom sites of the perfect crystal lattice. The superlattice Brillouin-zone sampling is tested by using a k-point mesh up to 64 points. These tests also give an idea about the effects of the deeplevel dispersion due to the supercell approximation.

The results of the convergence tests with respect to the size of the supercell and the number of $\mathbf{k}$ points are presented in Fig. 2 in the case of the total energy of the neutral $\left(V_{\mathrm{As}} \mathrm{Si}_{\mathrm{Ga}}\right)^{0}$ complex. The total energy is shown as a function of the Si displacement from the substitutional position along 
the [111] direction. The host atoms are not allowed to relax from their perfect lattice positions in these calculations. The zero of the energy scale is the total energy when the Si atom is in the substitutional site. Calculations are performed with supercells having 32 and 64 atoms. The Brillouin-zone sampling consists of the $\Gamma$-point calculations for both supercell sizes, for the 32-atom site supercell the Monkhorst-Pack ${ }^{14}$ $2 \times 2 \times 2 \mathbf{k}$-point mesh is used, and for the 64-atom site supercell the $2 \times 2 \times 2$ and $4 \times 4 \times 4$ Chadi-Cohen ${ }^{15}$ k-point meshes are employed. It can be seen that the calculations using several special $\mathbf{k}$ points give very similar results irrespective of the size of the supercell and the number of $\mathbf{k}$ points. In contrast, the use of the $\Gamma$ point in the Brillouinzone sampling gives total-energy curves which deviate remarkably from the special k-point results. Moreover, there is a large difference between the 32- and 64-atom-site supercells, so that the total-energy curve obtained with the larger supercell is closer to the results from the special k-point calculations. The prominent feature of the $\Gamma$-point calculations is that the total energy for the interstitial ionic configuration is low in comparison with that for the substitutional ionic configuration. This notion is in accord with the results by Furthmüller and Fähnle, ${ }^{16}$ who considered the possibility of the metastability of chalcogen impurities in $\mathrm{Si}$. In the case of the (neutral) As antisite (EL2 center) in an otherwise perfect GaAs lattice, we find that the total-energy difference between the interstitial and substitutional configurations is lowered when one uses special $\mathbf{k}$ points instead of the $\Gamma$-point, but the qualitative features presented, e.g. in Ref. 1, do not change.

In conclusion, the total-energy results using the $\Gamma$ point only are not fully converged even in the case of the 64-atomsite supercell, whereas the results obtained with the Monkhorst-Pack $2 \times 2 \times 2$ k-point mesh and the 32 -atom-site supercell are already quite well converged. However, due to the large defect-state dispersion, definite conclusions about the relative ordering of the two minima in the total-energy curves are difficult to give. The defect level dispersion is estimated to be of the order of $0.4 \mathrm{eV}$ for the larger cell. This estimate is based on the maximum difference between the eigenvalues calculated using the $4 \times 4 \times 4$ k-point mesh.

Next we allowed the ions to relax in the substitutional and interstitial configurations for the Si impurity, and studied the convergence of the ionic relaxations. We compared the nearest-neighbor relaxations from a calculation with the 32atom supercell with the Monkhorst-Pack $2 \times 2 \times 2$ k-point mesh to the ones obtained with the 64-atom supercell and the Chadi-Cohen $2 \times 2 \times 2$ k-point mesh. The maximum difference between these results corresponds to about $3 \%$ in the breathing mode relaxation. We did not perform symmetryunrestricted lattice relaxations for the 64-atom supercell and the $4 \times 4 \times 4$ k-point mesh because the computational cost becomes too high. Now having an idea about the convergence of the results with respect to the supercell size and the number of $\mathbf{k}$ points, in the following we report results only from calculations employing the 32-atom supercell and the $2 \times 2 \times 2$ k-point mesh.

We calculate the positron states and annihilation characteristics using the atomic superposition method. ${ }^{17}$ The method employs a non-self-consistent electronic structure. The average electron density is not affected by the presence
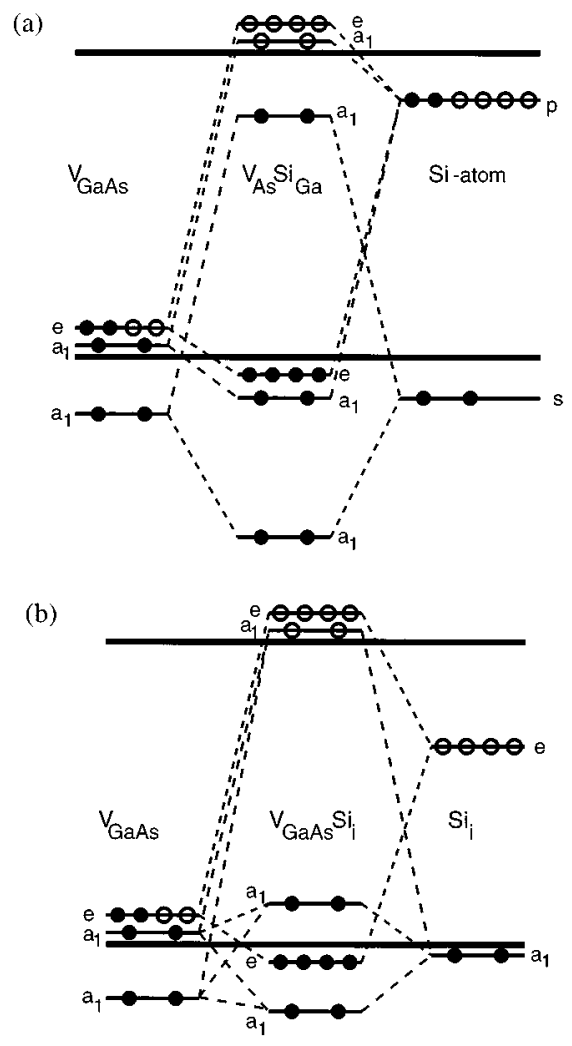

FIG. 3. Schematic figure of the linear combination of atomic

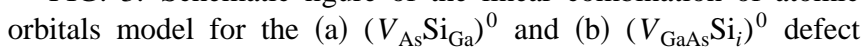
complexes.

of the positron, but the short-range electron pileup at the positron is taken into account in constructing the positron potential and in calculating the annihilation rate. In practice, for the electron-positron correlation effects, i.e., for the positron correlation potential and for the electron enhancement at the positron, we use the interpolation forms presented in Ref. 18 for the limit of the vanishing positron density. The method predicts especially well the changes in the positron lifetimes between the different systems (e.g., a perfect bulk lattice and a vacancy) in a reasonable agreement with experiments. ${ }^{19}$ Therefore we scale below the lifetime results, so that the calculated positron lifetime for the perfect bulk lattice coincides with the experimental one. The relative core-annihilation parameter is estimated from the annihilation rates with core and valence electrons as in Ref. 20, without calculating the actual momentum density of the annihilating electron-positron pairs. The total energy of the defect with a positron is estimated as the sum of the purely electronic energy of the system and the positron energy eigenvalue. ${ }^{21}$

\section{TIGHT-BINDING MODEL FOR DEEP LEVELS}

The appearance of the local-energy minimum for the donor impurity or the As antisite in the interstitial region near the As vacancy can be understood qualitatively by a simple tight-binding picture. In Fig. 3 we discuss the $V_{\mathrm{As}} \mathrm{Si}_{\mathrm{Ga}}$ defect, but the model applies equally well for $V_{\mathrm{As}} \mathrm{As}_{\mathrm{Ga}}$, the changes being merely in the positions of the deep states in the gap. The model is a modification of the one by Dabrowski and 
Scheffler ${ }^{1}$ for the EL2 centers and it is based on the hybridization of the divacancy $\left(V_{\mathrm{GaAs}}\right)$ states with the free $\mathrm{Si}$ atom or the interstitial $\mathrm{Si}$ impurity $\left(\mathrm{Si}_{i}\right)$ states corresponding to the

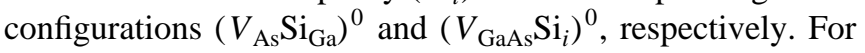
the divacancy the deep states are localized at the gallium vacancy end, and the electronic structure of the divacancy is quite close to the one of the isolated gallium vacancy. ${ }^{22}$ The effect of taking the As atom away from the lattice site neighboring the $D X$ (EL2) center should thus be rather small.

The divacancy states can be considered to result from the hybridization of the arsenic and gallium vacancy states. ${ }^{22,23}$ The divacancy has the same point symmetry of $C_{3 v}$ as the defect complexes discussed in this work. The divacancy has two deep states ( $e$ and $a_{1}$ ) in the band gap just above the valence-band maximum (VBM) (see Fig. 3). For the neutral divacancy both states are occupied by two electrons. The deep states of the divacancy originate from the splitting of the Ga-vacancy $t_{2}$ state due to the symmetry lowering from $T_{d}$ to $C_{3 v}$. The $e$ and $a_{1}$ states originating from the deep $t_{2}$ state of the As vacancy are empty resonance states in the conduction band. Below the VBM there exists $a_{1}$ states corresponding to the As and $\mathrm{Ga}$ vacancies.

In the substitutional configuration the $p$ orbitals of the $\mathrm{Si}$ atom hybridize with the divacancy $e$ and upper $a_{1}$ states resulting in $e$ and $a_{1}$ states from which the upper, antibonding ones are unoccupied resonance states in the conduction band, and the lower, bonding states lie in the valence band [see Fig. 3(a)]. The complex introduces one gap state, namely, an $a_{1}$ state, which results from the hybridization of the $a_{1}$ state of the divacancy (corresponding to the Ga vacancy end) with the $s$ orbital of the $\mathrm{Si}$ atom. This state has antibonding character between the $\mathrm{Si}$ atom and the neighboring As atoms. The effect of this state is to push the Si atom toward the arsenic vacancy.

In the interstitial configuration the states are considered to be formed by the hybridization of the states of the interstitial Si impurity and the divacancy states as shown in Fig. 3(b). The Si interstitial has an unoccupied $e$ state approximately in the middle of the band gap and two $a_{1}$ states, slightly below the VBM. The $e$ and the upper $a_{1}$ states originate from the $p$ orbitals of the $\mathrm{Si}$ atom, whereas the lower $a_{1}$ state comes from the $s$ orbital of the $\mathrm{Si}$ atom. The complex again introduces one gap state which differs from the one in the substitutional ionic configuration. The deep state, which has an antibonding character between the $\mathrm{Si}$ and $\mathrm{As}$ atoms, originates from the hybridization of the higher $a_{1}$ state of Si with the $a_{1}$ states of the divacancy. The effect of this state is to push the Si atom toward the open [111] direction.

The transition from the substitutional to the interstitial configuration does not involve symmetry lowering as in the case of the $D X$ centers $^{2}$ or the EL2 center. ${ }^{1}$ When the $\mathrm{Si}$ impurity or the $\mathrm{As}_{\mathrm{Ga}}$ antisite is moved from the substitutional site toward the interstitial [111] direction, an energy-level crossing of the $a_{1}$ states of the substitutional and interstitial configurations in the band gap takes place. This level crossing is reflected, in a fashion similar to the LLR model for the EL2 center, as a local maximum or energy barrier in the total energy, and as a local-energy minimum in the interstitial configuration.

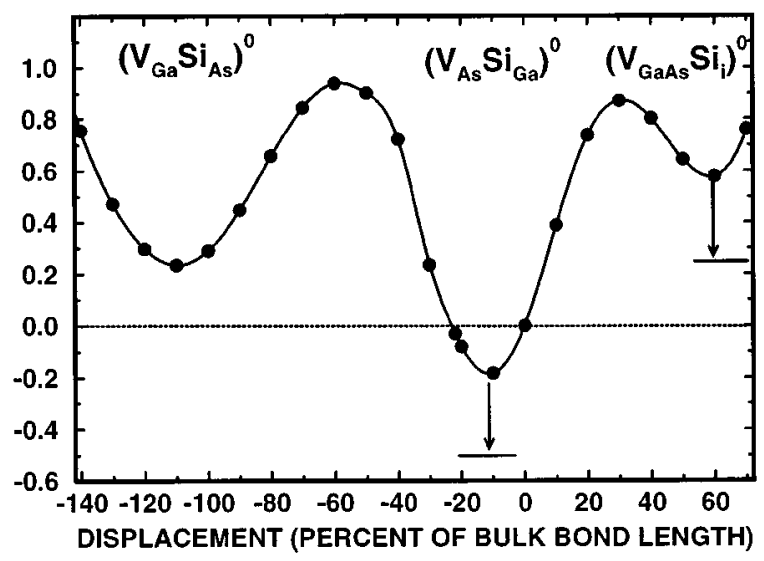

FIG. 4. Total energy of the neutral vacancy-impurity system as a function of the Si-atom displacement from the Ga-atom substitutional position. The zero of the energy corresponds to the total energy of the substitutional configuration. The arrows and horizontal lines show the energy lowering due to the atomic relaxations. The atomic relaxations conserve the $C_{3 v}$ symmetry of the defect.

\section{TOTAL-ENERGY CURVES}

The total energies of the defect complexes $\left(V_{\mathrm{As}} \mathrm{Si}_{\mathrm{Ga}}\right)^{0}$ and $\left(V_{\mathrm{As}} \mathrm{As}_{\mathrm{Ga}}\right)^{+}$are given in Figs. 4 and 5 as a function of the displacement of the Si or As atom in the [111] direction from the substitutional Ga-atom position. In these calculations all other atoms of the systems are kept at their ideal lattice positions. Actually, the ionic configurations mean that the displacement of the given atom with respect to an ideal divacancy $\left(V_{\mathrm{GaAs}}\right)$ is considered. The total-energy curves in Figs. 4 and 5 start from the configuration in which either a Si or As atom occupies a site beyond the As-vacancy end of the divacancy. This means that the latter case corresponds to a slightly deformed ideal Ga vacancy. The curves in Figs. 4 and 5 extend far into the interstitial region only from the As-vacancy end of the divacancy, because in the opposite direction (in the interstitial region near Ga atoms) we have not found a local-energy minimum. This finding is in accord with the calculations for $D X$ centers corresponding to the

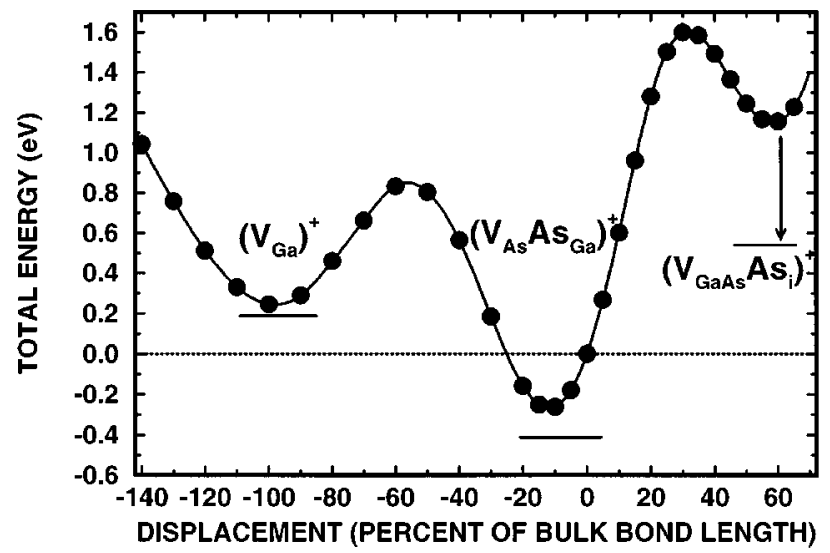

FIG. 5. Total energy of the vacancy-antisite system as a function of As-atom displacement from the Ga-atom substitutional position. The zero of the energy corresponds to the total energy of the Asvacancy-As-antisite configuration. The arrow and horizontal lines show the energy lowering due to the atomic relaxations. The atomic relaxations conserve the $C_{3 v}$ symmetry of the defect. 
anion and cation site donors: in both cases the LLR takes place via the movement of an atom (a cation site impurity or a Ga atom) to the interstitial region near As atoms. ${ }^{2}$

In Figs. 4 and 5 we show results only for the charge states in which the deep levels are occupied by two electrons. Supercell-DFT-LDA calculations do not give reliable total energies for the charge states in which the upper $a_{1}$ state is occupied by one or two electrons, because the Coulomb repulsion raises this state above the bottom of the LDA conduction band. This state is delocalized, and the supercell approximation does not properly describe an isolated defect. ${ }^{24}$ Similarly, if the deep levels are occupied by only one electron, the hole state may drop into the valence band. This happens for $\left(V_{\mathrm{GaAs}} \mathrm{As}_{i}\right)^{2+}$ and $\left(V_{\mathrm{GaAs}} \mathrm{Si}_{i}\right)^{+}$in the interstitial configurations. The raise of a deep level to the conduction band may be an artifact of the too narrow LDA band gap, and in reality there may be more charge states than it is possible to calculate accurately within the LDA. These charge states can be formally calculated, but the results will be only qualitative.

In the case of the Si impurity in the neutral charge state, the total-energy minimum corresponds to the pair $\left(V_{\mathrm{As}} \mathrm{Si}_{\mathrm{Ga}}\right)^{0}$. Actually, at the minimum the $\mathrm{Si}$ atom is slightly moved from the substitutional site toward the center of the As vacancy. The configuration $\left(V_{\mathrm{Ga}} \mathrm{Si}_{\mathrm{As}}\right)^{0}$ is $\sim 0.4 \mathrm{eV}$ higher in energy. The interstitial configuration $\left(V_{\mathrm{GaAs}} \mathrm{Si}_{i}\right)^{0}$ is $\sim 0.7$ $\mathrm{eV}$ higher in energy than the minimum-energy configuration. Atomic relaxation lowers the total energy by the same amount $(\sim 0.3 \mathrm{eV})$ both in the substitutional and interstitial configurations. For the interstitial configuration the displaced atom stays in the interstitial position indicating that the corresponding total-energy minima is not destroyed by relaxation.

For the As atom displaced along the [111] direction, the configuration corresponding to the positive Ga vacancy is in fact not stable because the lowest unoccupied level merges into the valence band and becomes occupied. This has been already discussed by Baraff and Schlüter. ${ }^{25}$ For the configurations corresponding to $\left(V_{\mathrm{As}} \mathrm{As}_{\mathrm{Ga}}\right)^{+}$and $\left(V_{\mathrm{GaAs}} \mathrm{As}_{\mathrm{i}}\right)^{+}$the lowest unoccupied level rises to the band gap stabilizing these configurations. However, in contrast to the results of Baraff and Schlüter, for the positive defect we do not find a metastable configuration corresponding to the position of the As atom between the As and Ga vacancy ends of the divacancy. The substitutional configuration $\left(V_{\mathrm{As}} \mathrm{As}_{\mathrm{Ga}}\right)^{+}$has the lowest energy and the interstitial configuration $\left(V_{\mathrm{GaAs}_{s}} \mathrm{As}_{i}\right)^{+}$ is about $\sim 1.35 \mathrm{eV}$ higher in energy. Atomic relaxation does not lower appreciably the total energy for the configurations corresponding to the positive Ga vacancy or $\left(V_{\mathrm{As}} \mathrm{As}_{\mathrm{Ga}}\right)^{+}$, but for the interstitial configuration the relaxation energy is substantial $(\sim 0.6 \mathrm{eV})$. The displaced As atom stays in the interstitial position, and the total-energy difference with respect to the minimum energy configuration is reduced to $\sim 1.0 \mathrm{eV}$. We have not used any symmetry constraint during the relaxations, but the symmetry of these defects $\left(C_{3 v}\right)$ is conserved during the relaxation process.

It is interesting to compare the electron-density distribution of the occupied deep state for the As-vacancy-Siimpurity or As-vacancy-As-antisite complex in interstitial configurations with those for the isolated $D X$ or EL2 centers in the LLR model. The deep-state density ${ }^{2}$ for the $D X$ center

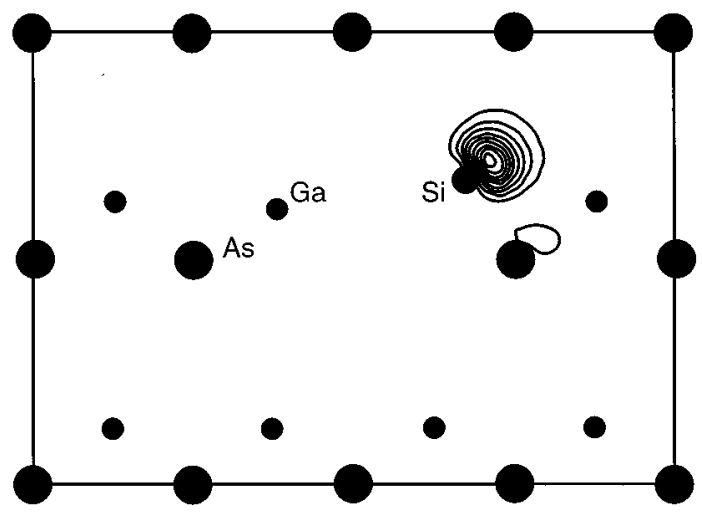

FIG. 6. Electron density associated with the deep state of $\left(V_{\mathrm{GaAs}} \mathrm{Si}_{i}\right)^{0}$. The contour spacing is 1.0 electrons per bulk GaAs unit cell volume.

is in fact quite close to that shown in Fig. 6 for the Asvacancy-Si-impurity complex. For example, in both cases the maximum electron density lies between the $\mathrm{Si}$ atom and the tetrahedral interstitial site in the [111] direction. In contrast, the deep-state density ${ }^{26}$ for the EL2 center shows a strong dangling bond near the As atom left behind. In the case of the As-vacancy-As-antisite complex this maximum is missing and the density distribution resembles closely that for the As-vacancy-Si-impurity complex. A comparison of isolated centers with vacancy-impurity or antisite complexes, and the existence of local total-energy minima for complexes in interstitial configurations, shows that the interstitial configurations are stabilized to a large extent by the repulsion between the impurity or antisite atom and the three neighboring As atoms in the case of isolated EL2 and $D X$ centers. Due to the different characters of the wave functions, this contribution to the stabilization is larger in the case of the $\mathrm{Si}$ impurity than the As antisite, so that the interstitial configuration is relatively more stable for the $\mathrm{Si}$ impurity.

An important question connected to the existence of the metastability is the mechanism of possible transitions between the different energy minima. In the case of the EL2 center, Dabrowski and Scheffler ${ }^{26}$ showed that the excitation of one electron from the doubly occupied $a_{1}$ deep level to the lowest unoccupied $a_{1}$-type deep level may cause a transition from a stable substitutional state to an interstitial state higher in energy. In a corresponding calculation employing only the $\Gamma$ point, we found that an excited state with a similar behavior of the total energy also exists for the Asvacancy-As-antisite pair. Thus it could be possible to excite the defect pair into the interstitial configuration optically. Furthermore, in accord with the results for the isolated EL2 and $D X$ centers, ${ }^{26}$ our partly qualitative (see the discussion of the charge states above) calculations show that adding or removing one or more electrons from $\left(V_{\mathrm{As}} \mathrm{Si}_{\mathrm{Ga}}\right)^{0}$ or $\left(V_{\mathrm{As}} \mathrm{As}_{\mathrm{Ga}}\right)^{+}$strongly increases the total energy of the interstitial configuration relative to the substitutional one. Thus the change of the charge state could induce regeneration from a metastable interstitial configuration to a substitutional one. As a matter of fact, in the case of neutral and negative charge states of the As atom within the divacancy, we have found that the lowest-energy configuration corresponds to the neutral or negative Ga vacancy; i.e., we have reproduced 


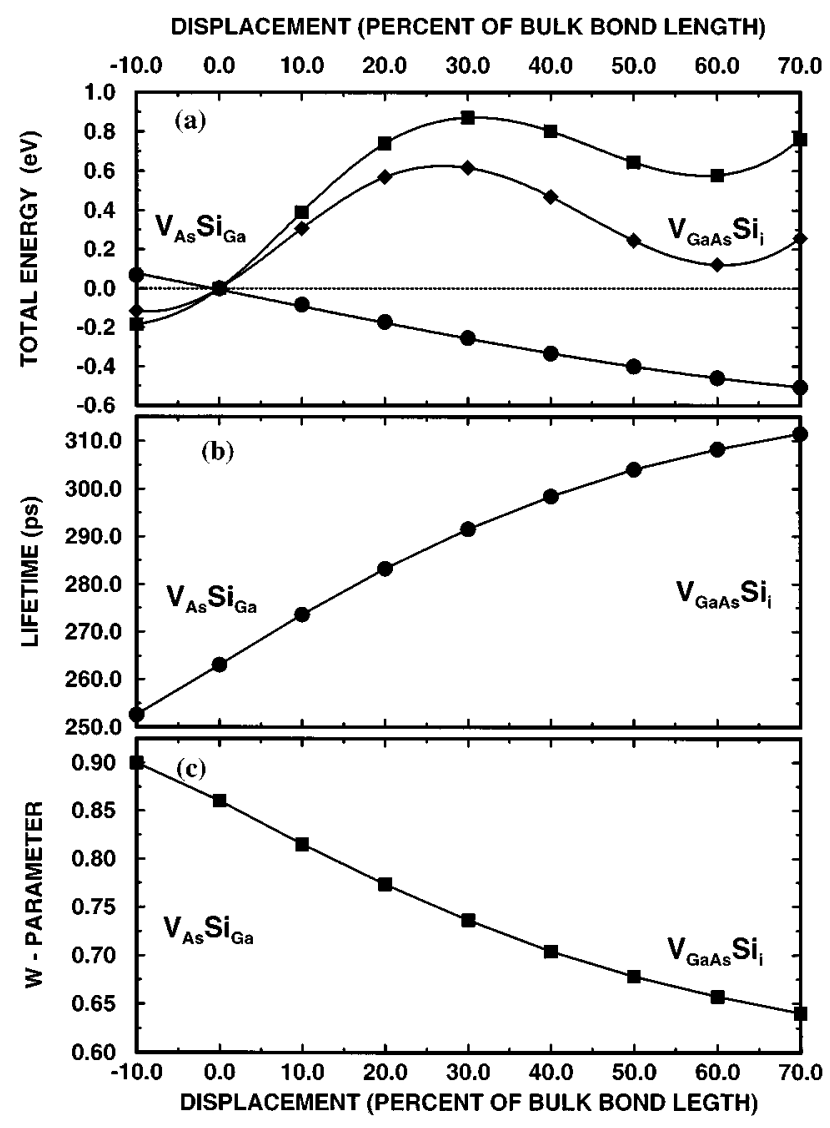

FIG. 7. Characteristics of the positron trapped by a neutral vacancy-impurity system as a function of the $\mathrm{Si}$ atom displacement from the substitutional position along the open [111] direction. (a) The change in the total energy of the electronic system (squares), positron energy eigenvalue (circles) and their sum (diamonds). (b) The positron lifetime and (c) relative core annihilation parameter $\mathrm{W}$ as a function of silicon atom displacement. Other atomic relaxations from the ideal lattice positions have not been allowed.

the bistability of the defect between configurations $\left(V_{\mathrm{As}} \mathrm{As}_{\mathrm{Ga}}\right)^{+}$and $V_{\mathrm{Ga}}^{0} \cdot{ }^{25}$

\section{POSITRON ANNIHILATION CHARACTERISTICS}

The results of calculations for the positron state and annihilation characteristics are shown in Fig. 7 in the case of the As-vacancy-Si-impurity pair. The calculations are performed as a function of the position of the $\mathrm{Si}$ atom, and all other atoms are kept in their ideal lattice positions. The increase of the open volume lowers the positron energy eigenvalue, as shown in Fig. 7(a). When the positron energy is added to the electronic lattice energy (cf. Fig. 2), their sum in the interstitial configuration is only $\sim 0.2 \mathrm{eV}$ higher than in the substitutional configuration. The positron lifetime in Fig. 7(b) is scaled so that the calculated lifetime for the perfect bulk lattice coincides with the experimental lifetime of 231 ps. ${ }^{7}$ The positron lifetime increases with the increase of the open volume, and shows a slight saturation with the distance of the $\mathrm{Si}$ atom from the As vacancy. The relative positron core-annihilation parameter $W$ (Ref. 20) (scaled so that, for bulk GaAs, $W=1$ ) given in Fig. 7 shows that the coreannihilation contribution to the total annihilation rate de-
TABLE I. Theoretical positron lifetimes $\tau$ and $W$ parameters for the ideal (unrelaxed) vacancies and the divacancy in GaAs. The line-shape parameter is scaled so that for bulk GaAs, $W=1$.

\begin{tabular}{ccc}
\hline \hline Vacancy & $\tau(\mathrm{ps})$ & $W$ \\
\hline Bulk & 231 & 1.00 \\
$V_{\mathrm{Ga}}$ & 264 & 0.69 \\
$V_{\mathrm{As}}$ & 261 & 0.96 \\
$V_{\mathrm{GaAs}}$ & 317 & 0.59 \\
\hline \hline
\end{tabular}

creases with the increasing open volume.

The calculated positron annihilation characteristics for the As-vacancy-Si-impurity complex can be compared with those for the ideal As and Ga vacancies and the ideal divacancy in GaAs given in Table I. The positron lifetimes for the ideal As and Ga vacancies are nearly the same, and, according to Fig. 7(b), the lifetime for the As vacancy is nearly unaffected if one of the nearest-neighbor Ga atoms is substituted with a Si impurity. The positron lifetime for the ideal divacancy is also clearly longer than that for the Asvacancy-Si-impurity complex when the $\mathrm{Si}$ atom is in an interstitial configuration corresponding to the local-energy minimum. The estimated $W$ parameter for the ideal Ga vacancy is remarkably smaller than that for the ideal As vacancy. The reason is that annihilation with the $\mathrm{Ga} 3 d$ electrons neighboring the As vacancy is considerably stronger than the annihilation with the more localized As $3 d$ electrons neighboring the Ga vacancy. The substitution of a $\mathrm{Ga}$ atom with a $\mathrm{Si}$ atom at the As vacancy clearly lowers the $W$ parameter because of the smaller core of the $\mathrm{Si}$ atom. This shows that the $W$ parameter is a more sensitive parameter for the chemical environment than the positron lifetime. The $W$ parameter for the ideal divacancy is seen to be only slightly smaller than that for the As-vacancy-Si-impurity complex when the $\mathrm{Si}$ atom is in the interstitial configuration.

It is interesting to compare the predicted positron annihilation characteristics with those measured for defects in GaAs. According to the measurements for electron-irradiated semi-insulating GaAs, the positron lifetime for the $\mathrm{Ga}$ vacancy is 260 ps. $^{7}$ The $W$ parameter measured by the Doppler-broadening technique for the Ga vacancy is $0.74 .^{20}$ These numbers are in good agreement with the theoretical estimates for the ideal Ga vacancy. Saarinen et al. ${ }^{7}$ found two different positron lifetimes of 257 and 295 ps, which they assigned to As-vacancy defects in GaAs. The former is identified as a singly negative charge state, and the latter as a neutral charge state of the defect. Saarinen et al. ${ }^{7}$ emphasized that the As vacancy they saw may be isolated or bound to a defect complex. The large lifetime change indicates a large change in the ionic relaxations, such that the open volume seen be the positron decreases strongly when the neutral vacancy captures an electron. For example, if this lifetime change is associated in a theoretical calculation with a symmetry-conserving breathing mode relaxation, all $\mathrm{Ga}$ atoms neighboring the As vacancy should move about $10 \%$ of the bond distance in GaAs. ${ }^{27}$ The shorter lifetime of $257 \mathrm{ps}$ is close to that estimated for the ideal As vacancy (Table I). The longer lifetime of $295 \mathrm{ps}$ is close to the average between the monovacancy and divacancy lifetimes. The dependence of the positron lifetime on the charge state of the vacancy 
defect has also been seen in $\mathrm{Si}^{28}$

Considering the theoretical positron annihilation characteristics in Fig. 7 and in Table I, the As vacancy and Siimpurity in the interstitial configuration represent an open volume which is between the ideal monovacancy and the ideal divacancy. Thus one could consider the defect as "one and a half vacancies.' Furthermore, the EL2 and $D X$ centers in their interstitial atomic configurations can be thought to obtain a Ga vacancy, but the open volume is less than that for an ideal vacancy, and the term "half a vacancy" is appropriate for these defects. As a matter of fact, these defects can, according to experiments, trap positrons, and the positron lifetime or core-annihilation parameter for these defects is between the values for the perfect bulk and the ideal vacancy.

\section{CONCLUSIONS}

We have studied the energetics of defect complexes formed by an As vacancy and the cation-site donor ( $\mathrm{Si}$ ) and the antisite $\mathrm{As}_{\mathrm{Ga}}$ in GaAs. In charge states with two deep electrons in the band gap $\left[\left(V_{\mathrm{As}} \mathrm{Si}_{\mathrm{Ga}}\right)^{0}\right.$ and $\left.\left(V_{\mathrm{As}} \mathrm{As}_{\mathrm{Ga}}\right)^{+}\right]$, total energies show two minima as a function of the position of the dopant or the antisite atom along the [111] direction from the substitutional to interstitial site close to three As atoms. The behavior of the total energy therefore resembles that of the EL2 and $D X$ centers in the large-lattice-relaxation model, although one neighboring As atom is missing. The interstitial atomic configuration is stabilized by the repulsion due to the antibonding states between the displaced atom and three As atoms. This stabilization mechanism should also contribute in the case of an isolated $D X$ center.

The total energy corresponding to the interstitial configuration is found to be higher than that for the substitutional nearest-neighbor defect configuration. The change in the charge state caused by removing electrons from or adding to the deep states raises the energy of the interstitial configuration relative to the substitutional one. The drawing of definitive conclusions about the relative ordering of the energy minima is, however, difficult. This is due to the dispersion of the deep levels in the supercell method. For example, we have shown that the use of only the $\Gamma$ point in the superlattice-Brillouin-zone sampling leads to a strong supercell-size dependence of the total energy. The use of special $\mathbf{k}$ points leads to a better convergence for small supercell sizes. In any case the calculations give strong evidence of the existence of the metastability of vacancyimpurity and vacancy-antisite pairs.

We have calculated positron states and annihilation characteristics for the neutral $V_{\mathrm{As}} \mathrm{Si}_{\mathrm{Ga}}$ defect as a function of the position of the $\mathrm{Si}$ atom. The addition of the positron to the defect lowers the total energy of the interstitial configuration close to that of the substitutional configuration. The positron lifetime and core-annihilation parameters for the substitutional configuration are similar to those for the ideal monovacancy, whereas the annihilation characteristics for the interstitial configuration lie between those for the monovacancy and the divacancy.

\section{ACKNOWLEDGMENTS}

The authors wish to thank Professor P. Hautojärvi and Dr. K. Saarinen for many valuable discussions. One of us (S.P.) acknowledges partial financial support by the Jenny and Antti Wihuri Foundation. This research has been supported by a MATRA grant from the Academy of Finland. We also acknowledge the generous computing resources of the Center for Scientific Computing (CSC).
*Electronic address: Sami.Poykko@ hut.fi

†Electronic address: Martti.Puska@hut.fi

†Electronic address: Matti.Alatalo@hut.fi

${ }^{\S}$ Electronic address: Risto.Nieminen@hut.fi

${ }^{1}$ D. J. Chadi and K. J. Chang, Phys. Rev. Lett. 61, 2187 (1988); J. Dabrowski and M. Scheffler, ibid. 60, 2183 (1988).

${ }^{2}$ D. J. Chadi and K. J. Chang, Phys. Rev. B 39, 10063 (1989).

${ }^{3}$ See, for example, D. C. Look, Z. Q. Fang, and J. R. Sizelove, Phys. Rev. B 49, 16757 (1994); F. D. Auret, S. A. Goodman, and W. E. Meyer, Appl. Phys. Lett. 67, 3277 (1995).

${ }^{4}$ M. Hesse, F. K. Koschnick, K. Krambrock, and J.-M. Spaeth, Solid State Commun. 92, 207 (1994).

${ }^{5}$ R. Krause, K. Saarinen, P. Hautojärvi, A. Polity, G. Gärtner, and C. Corbel, Phys. Rev. Lett. 65, 3329 (1990).

${ }^{6}$ J. Mäkinen, T. Laine, K. Saarinen, P. Hautojärvi, C. Corbel, V. M. Airaksinen, and P. Gibart, Phys. Rev. Lett. 71, 3154 (1993).

${ }^{7}$ K. Saarinen, P. Hautojärvi, P. Lanki, and C. Corbel, Phys. Rev. B 44, 10585 (1991); C. Corbel, M. Stucky, P. Hautojärvi, K. Saarinen, and P. Moser, ibid. 38, 8192 (1988).

${ }^{8}$ See, for example, M. C. Payne, M. P. Teter, D. C. Allan, T. A. Arias, and J. D. Joannopoulos, Rev. Mod. Phys. 64, 1045 (1992); R. Car and M. Parrinello, Phys. Rev. Lett. 55, 2471 (1985).
${ }^{9}$ D. M. Ceperley and B. J. Alder, Phys. Rev. Lett. 45, 566 (1980); J. Perdew and A. Zunger, Phys. Rev. B 23, 5048 (1981).

${ }^{10}$ G. B. Bachelet, D. R. Hamann, and M. Schlüter, Phys. Rev. B 26, 4199 (1982); R. Stumpf, X. Gonze, and M. Scheffler (unpublished).

${ }^{11}$ L. Kleinman and D. M. Bylander, Phys. Rev. Lett. 48, 1425 (1982).

${ }^{12}$ F. Tassoni, F. Mauri, and R. Car, Phys Rev. B 50, 10561 (1994).

${ }^{13}$ A. R. Williams and J. Soler, Bull. Am. Phys. Soc. 32, 562 (1987).

${ }^{14}$ H. J. Monkhorst and J. D. Pack, Phys. Rev. B 13, 5188 (1976).

${ }^{15}$ D. J. Chadi and M. L. Cohen, Phys. Rev. B 8, 5747 (1973).

${ }^{16}$ J. Furthmüller and M. Fähnle, Phys. Rev. B 46, 3839 (1992).

${ }^{17}$ M. J. Puska and R. M. Nieminen, J. Phys. F 13, 333 (1983); A. P. Seitsonen, M. J. Puska, and R. M. Nieminen, Phys. Rev. B 51, 14057 (1995).

${ }^{18}$ E. Boronski and R. M. Nieminen, Phys. Rev. B 34, 3820 (1986).

${ }^{19}$ B. Barbiellini, M. J. Puska, T. Korhonen, A. Harju, T. Torsti, and R. M. Nieminen, Phys. Rev. B 53, 16201 (1996).

${ }^{20}$ M. Alatalo et al., Phys. Rev. B 54, 2397 (1996).

${ }^{21}$ M. J. Puska, A. P. Seitsonen, and R. M. Nieminen, Phys. Rev. B 52, 10947 (1995)

${ }^{22}$ S. Pöykkö, M. J. Puska, and R. M. Nieminen, Phys. Rev. B 53, 3813 (1996). 
${ }^{23}$ H. Xu, J. Appl. Phys. 72, 3522 (1992); Phys. Rev. B 46, 12251 (1992).

${ }^{24}$ For the $D X$ center in GaAs, see the discussion in M. Saito, A. Oshiyama, and O. Sugino, Phys. Rev. B 45, 13745 (1992).

${ }^{25}$ G. A. Baraff and M. Schlüter, Phys. Rev. Lett. 55, 2340 (1985).
${ }^{26}$ J. Dabrowski and M. Scheffler, Phys. Rev. B 40, 10391 (1989).

${ }^{27}$ S. Mäkinen and M. J. Puska, Phys. Rev. B 40, 12523 (1989).

${ }^{28}$ J. Mäkinen, P. Hautojärvi, and C. Corbel, J. Phys. Condens. Matter 4, 5137 (1992). 